\title{
Signal Amplification by Reversible Exchange for COVID-19 Antiviral Drug Candidates
}

Hye Jin Jeong ${ }^{1}$, Sein $\mathrm{Min}^{2}$, Heelim Chae ${ }^{2}$, Sara Kim², Gunwoo Lee ${ }^{1}$, Sung Keon Namgoong ${ }^{2}$, Keunhong Jeong ${ }^{1 *}$

${ }^{1}$ Department of Physics and Chemistry, Korea Military Academy, Seoul 01805, South Korea

${ }^{2}$ Department of Chemistry, Seoul Women's University, Seoul 01797, South Korea

\begin{abstract}
Several drug candidates have been proposed and tested as the latest clinical treatment for the coronavirus pneumonia (COVID-19). Chloroquine, hydroxychloroquine, ritonavir/lopinavir, and favipiravir are proved to be effective after treatment. The hyperpolarization technique presents an ability to further understand the roles of these drugs at the molecular scale and applications in nuclear magnetic resonance/magnetic resonance imaging (NMR/MRI). This technique may provide new opportunities in diagnosis and biomedical research to cope with COVID-19. Signal amplification by reversible exchange (SABRE)-based hyperpolarization studies on large-sized drug candidates were carried out. We observed hyperpolarized proton signals from whole structures, due to the unprecedented long-distance polarization transfer by para-hydrogen. We also found that the optimum magnetic field for the maximum polarization transfer yield was dependent on the molecular structure. Therefore, future research on isotope labelling and polarization transfer on long T1 time nuclei including clinical perspectives can help us overcome this worldwide pandemic.
\end{abstract}

Keywords: Chloroquine, Hydroxychloroquine, Ritonavir/Lopinavir, Favipiravir, SABRE, Hyperpolarization, NMR, COVID-19

\section{Corresponding Author}

* K. Jeong - Phone: +82-2-2197-2823, E-mail: doas1mind@kma.ac.kr 


\section{Introduction}

The recent coronavirus pneumonia (COVID-19) is a serious respiratory infectious disease. Patients with the coronavirus infection demonstrate fever with body temperatures exceeding $38^{\circ} \mathrm{C}$ and symptoms such as dry cough, fatigue, dyspnea, difficulty in breathing, and frosted glass-like symptoms in the lungs. ${ }^{1}$ The disease is known to be highly transmittable without severe symptoms. The number of cases has reached over a million worldwide.

To overcome this pandemic, many researchers are working to develop a vaccine. However, the vaccine is estimated to take over a year before it is available for public use. As a result, the global clinical community is attempting to repurpose existing drugs to tackle COVID-19. Recently, several drugs that can inhibit specific functions of the virus have been proposed and tested for the latest clinical treatments. Such drugs include chloroquine and hydroxychloroquine, which are more soluble, produce relatively less toxic metabolites, and have a definite effect on the novel coronavirus pneumonia. ${ }^{2}$ Chloroquine and hydroxychloroquine, utilized clinically over the last seventy years, are autoimmune disease drugs that are also antimalarial. The organic compounds have recently been reported as potential broad-spectrum antiviral drugs for COVID-19.3,4 From the molecular perspective, these drugs inhibit viral infection by increasing the endosomal $\mathrm{pH}$ required for virus and cell fusion and interfere with the glycosylation of cellular receptors of SARS-CoV. ${ }^{5}$ They also play a role in immune-modulating activity, potentially having a synergistic antiviral effect in vivo. ${ }^{6}$ Chloroquine is administered orally, upon which it distributes across the whole body, including the lungs. The EC90 value of chloroquine against 2019-nCoV in Vero E6 cells was $6.90 \mu \mathrm{M}$. This could be clinically achieved after $500 \mathrm{mg}$ of dosing.7,8 For COVID-19 treatment, a mega-dosage and/or dynamic distribution for an in vivo study under specific clinical conditions may be warranted.

Ritonavir/lopinavir, the combination drug marketed as Kaletra, is a relatively newly developed medication for the treatment and prevention of HIV. This compound has also been suggested as a potential drug candidate against COVID-19 because of its role as a proteinase inhibitor, inhibiting the polyprotein processing of Coronavirus. ${ }^{9,10}$ Recent reports have provided evidence in favor of this drug for the treatment of COVID-19. It acts to lessen viral loads and improve clinical symptoms. ${ }^{11,12}$ However, compared to 
chloroquine and hydroxychloroquine, the interactions of this drug in the body at the molecular scale have not been elucidated so far.

Favipiravir, which has been approved in Japan for the treatment of influenza since $2014^{13,14}$, has also shown effectiveness at accelerating viral clearance in the Chinese trials of hundreds of patients. ${ }^{15}$

To further understand drug interactions with proteins, their metabolism, and other activities, nuclear magnetic resonance (NMR) spectroscopy has been widely in pharmacokinetics. However, there is inherent insensitivity due to the small population differences in the spin state energy. The hyperpolarization technique, generating the nonBoltzmann distribution of the spin state population, may provide a breakthrough to address this challenge. Moreover, magnetic resonance imaging (MRI) poses as a solution to understand drug distribution throughout the body in vivo and study its real-time activity. To visualize the MRI real-time signal, the drug needs to be tagged with special materials, such as chelating agents with T1/T2 contrast. However, if additional materials are tagged in the drug candidate, it is likely to result in unpredictable effects from different molecular structures. The hyperpolarization technique can be applied to overcome this limitation as it promotes the visualization of the hyperpolarized signal through the MRI. Despite the need for this state-of-the-art technology to address several challenges, it presents itself as the key to visualize in vivo real-time distribution and activity to cope with COVID-19 in the future. It provides an opportunity to conduct detailed studies on the activity of antiviral drugs against COVID-19 in the body.

Of the several methods to hyperpolarize drugs, the para-hydrogen-based signal amplification by reversible exchange (SABRE) method is promising for the hyperpolarization of several key structures with nitrogen. In this method, the polarization may be transferred in real-time from protons to other isotopes such as ${ }^{13} \mathrm{C}^{16},{ }^{15} \mathrm{~N}^{17,18},{ }^{31} \mathrm{P}^{19},{ }^{19} \mathrm{~F}^{20},{ }^{119} \mathrm{Sn}^{21}$, and ${ }^{29} \mathrm{Si}^{22}$ without chemical changes.

Recently, several breakthrough studies developing hyperpolarized drugs or metabolites ${ }^{16-20}$, including those in pharmacokinetics, confirm that $\mathrm{SABRE}^{21}$ could have wide-scale application. This is particularly during a time when scientific solutions are the only way to conquer this pandemic. However, hyperpolarized drugs or metabolites are limited to small-sized molecules, constraining the practical applications for many studies. A hyperpolarization study using SABRE may serve a good and critical start to facilitate the future application of MRI scanning using hyperpolarized COVID-19 drug candidates, which mostly have large molecular weight. 
It is anticipated that SABRE-based hyperpolarization studies on such large molecules may enable the study of higher number of drugs and metabolites. To the best of our knowledge, this study is the first to evaluate SABRE-based hyperpolarization of specific COVID-19 drug candidates in real-time.

\section{Methods}

Favipiravir, hydroxychloroquine sulfate, and ritonavir were purchased from Shanghai Alkynechem Co., Ltd. (Shanghai, China) and were employed without further purifying. Chloroquine diphosphate salt (98.5\%) and lopinavir (98\%) were acquired from Sigma-Aldrich. Methanol- $d_{4}\left(\mathrm{CD}_{3} \mathrm{OD}, 99.8\right.$ atom \% D, Eurisotop) was used as received. ${ }^{1} \mathrm{H}$ NMR spectra employed for the characterization of favipiravir, chloroquine, hydroxychloroquine, lopinavir, and ritonavir were acquired on a Bruker Avance $\amalg$ NMR spectrometer operating at a ${ }^{1} \mathrm{H}$ resonance frequency of $300 \mathrm{MHz}$ and referenced to the residual $\mathrm{CH}_{3}$ peak of $\mathrm{CD}_{3} \mathrm{OD}(\delta=3.31)$. Hyperpolarization studies were conducted in the same manner. A home-built instrument designed as the para-hydrogen generator for hydrogen gas (a mixture of the spin isomers orthohydrogen and para-hydrogen) was conceived to go through a heat exchanger packed with a $\mathrm{FeO}(\mathrm{OH})$ catalyst (Sigma Aldrich) ${ }^{29}$ This structure was filled with liquid nitrogen in a Dewar flask and produced ca. 50\% para-hydrogen. In each experiment, para-hydrogen was continuously flowing into the chloroquine sample at a rate of $6 \mathrm{~mL} / \mathrm{min}$ at $23{ }^{\circ} \mathrm{C}$ and $1 \mathrm{~atm}$. To obtain the various magnetic field data, the following system was established and developed: The power supply was GPS-1850D (Bench Power Supply, Linear DC). A shielded coil wound with copper wire and a shielded coil outside the first consisted of a $200 \mathrm{~mm}$ diameter and 190 $\mathrm{mm}$ height. The magnetic field through the shielded coil was regulated by setting the current, which was in the range 0-5 $\mathrm{A}$.

The favipiravir to be polarized $\left(5 \mathrm{mg}, 3.1 \times 10^{-2} \mathrm{mmol}\right)$ and the pre-catalyst ([Ir(IMes)(COD)Cl], $\left.2 \mathrm{mg}, 3.1 \times 10^{-3} \mathrm{mmol}\right)$ were dissolved in $\mathrm{CD}_{3} \mathrm{OD}(900 \mu \mathrm{L})$. The samples of the hyperpolarization for chloroquine and hydroxychloroquine were prepared by treating a solution of substrate $\left(3.9 \times 10^{-3} \mathrm{mmol}\right)$ and the pre-catalyst $\left([\operatorname{Ir}(\mathrm{IMes})(\mathrm{COD}) \mathrm{Cl}], 2 \mathrm{mg}, 3.1 \times 10^{-3}\right.$ $\mathrm{mmol})$ in $\mathrm{CD}_{3} \mathrm{OD}(900 \mu \mathrm{L})$. In case of the lopinavir and ritonavir $\left(1.6 \times 10^{-2} \mathrm{mmol}\right)$ were added to the $\mathrm{CD}_{3} \mathrm{OD}(900 \mu \mathrm{L})$ solution of pre-catalyst ([Ir(IMes)(COD)Cl], $2 \mathrm{mg}, 3.1 \times 10^{-3} \mathrm{mmol}$ ). The mixture of favipiravir and the pre-catalyst was bubbled by para-hydrogen for $20 \mathrm{~min}$ for activation on the NMR tube. The mixture was analyzed in the NMR spectrometer, all NMR 
spectra were acquired with 1 scan in a varying magnetic field (Earth's magnetic field, 30 G, 50 G, $70 \mathrm{G}, 90 \mathrm{G}$, and $110 \mathrm{G}$, respectively). The diverse experiments of substrate were conducted in the same manner as mentioned above.

\section{Results and Discussion}

Favipiravir SABRE Favipiravir contains a pyrazine moiety, recognized as the polarization source in the SABRE mechanism. ${ }^{30}$ However, this complicated structure with pyrazine moiety was not applied for SABRE hyperpolarization (Figure 1).<smiles></smiles>

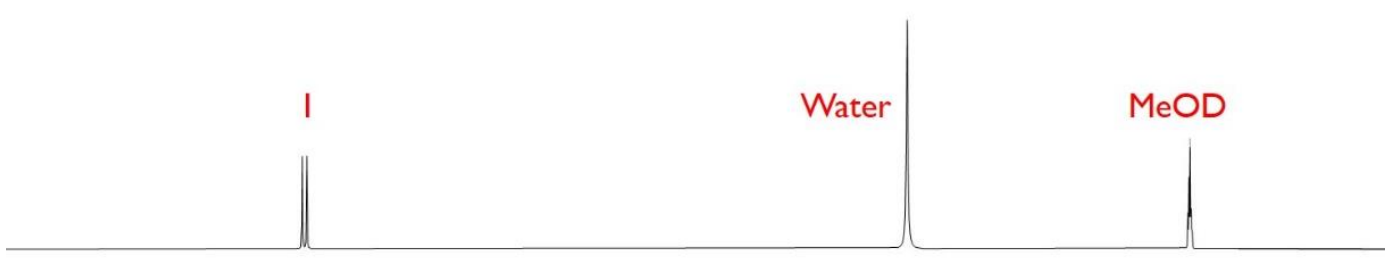

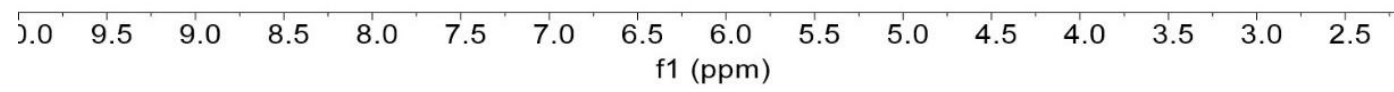

Figure 1. Favipiravir molecular structure and the normal NMR signal.

Figure 4 depicts an estimate of its complex with an Ir-catalyst, forming an exchangeable bond between $\operatorname{Ir}(\mathrm{I})$ and the pyrazine moiety. This form is expected for favipiravir to have transferred polarization from para-hydrogen. As predicted, we successfully obtained its hyperpolarized signal from the aromatic proton of favipiravir with a 20 fold enhanced signal after SABRE. An additional polarized signal around 6-7 ppm was observed and was attributed to protons in the hydroxy and amide groups in the structure. The SABRE-based polarization trend with 
magnetic field was maximized around $50 \mathrm{G}$, which is consistent with previous reports (Figure 2). ${ }^{31-33}$

a)<smiles>NC(=O)c1nc(F)cnc1O</smiles>
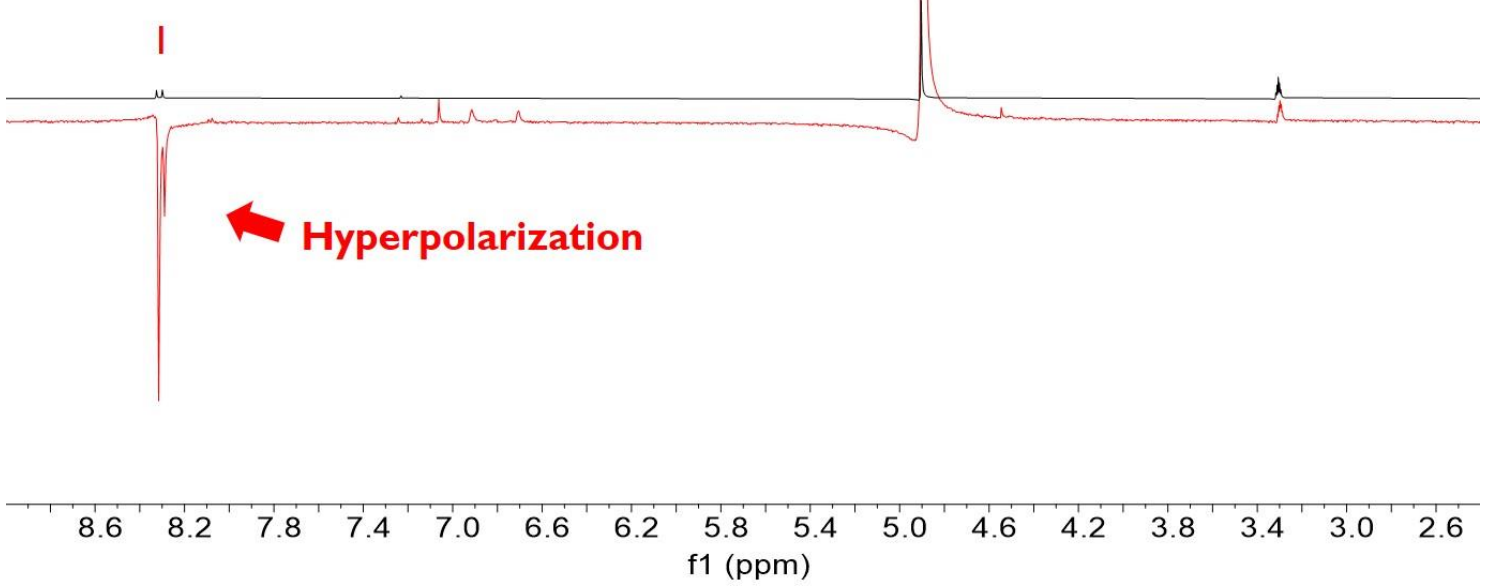

b)

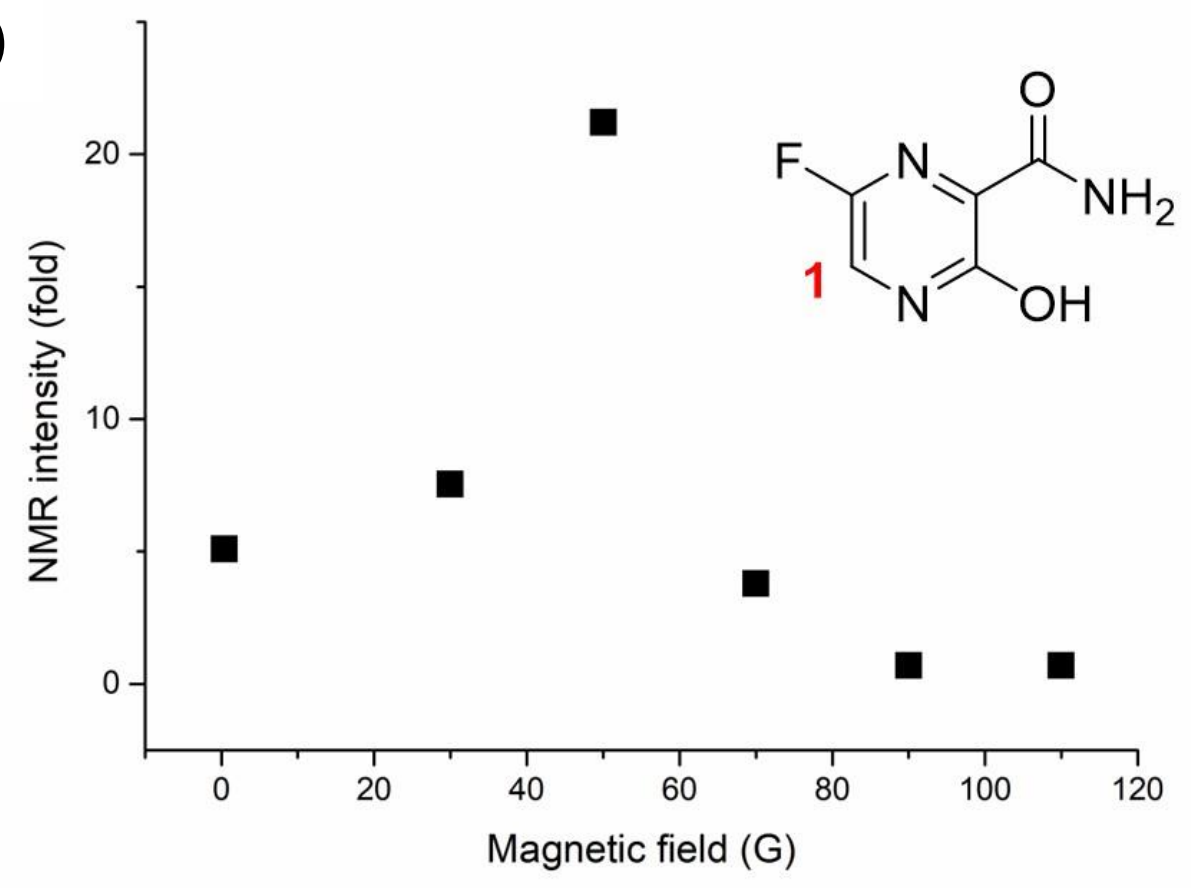


Figure 2. a) Hyperpolarized signals from favipiravir after SABRE in the presence of a $50 \mathrm{G}$ magnetic field; and b) Amplification number of $\mathrm{H}-1$ from the hyperpolarized favipiravir structure.

Chloroquine SABRE Chloroquine (hydroxychloroquine) contains a quinoline structure, which may potentially be hyperpolarized using SABRE. However, chloroquine has a long attachment (Figure 1), which could not be operable as SABRE is dependent on the chemical exchange reaction. It is assumed that transfer of its binding and kinetics to hyperpolarization with sufficient time from para-hydrogen may be challenging. (Figure 3).

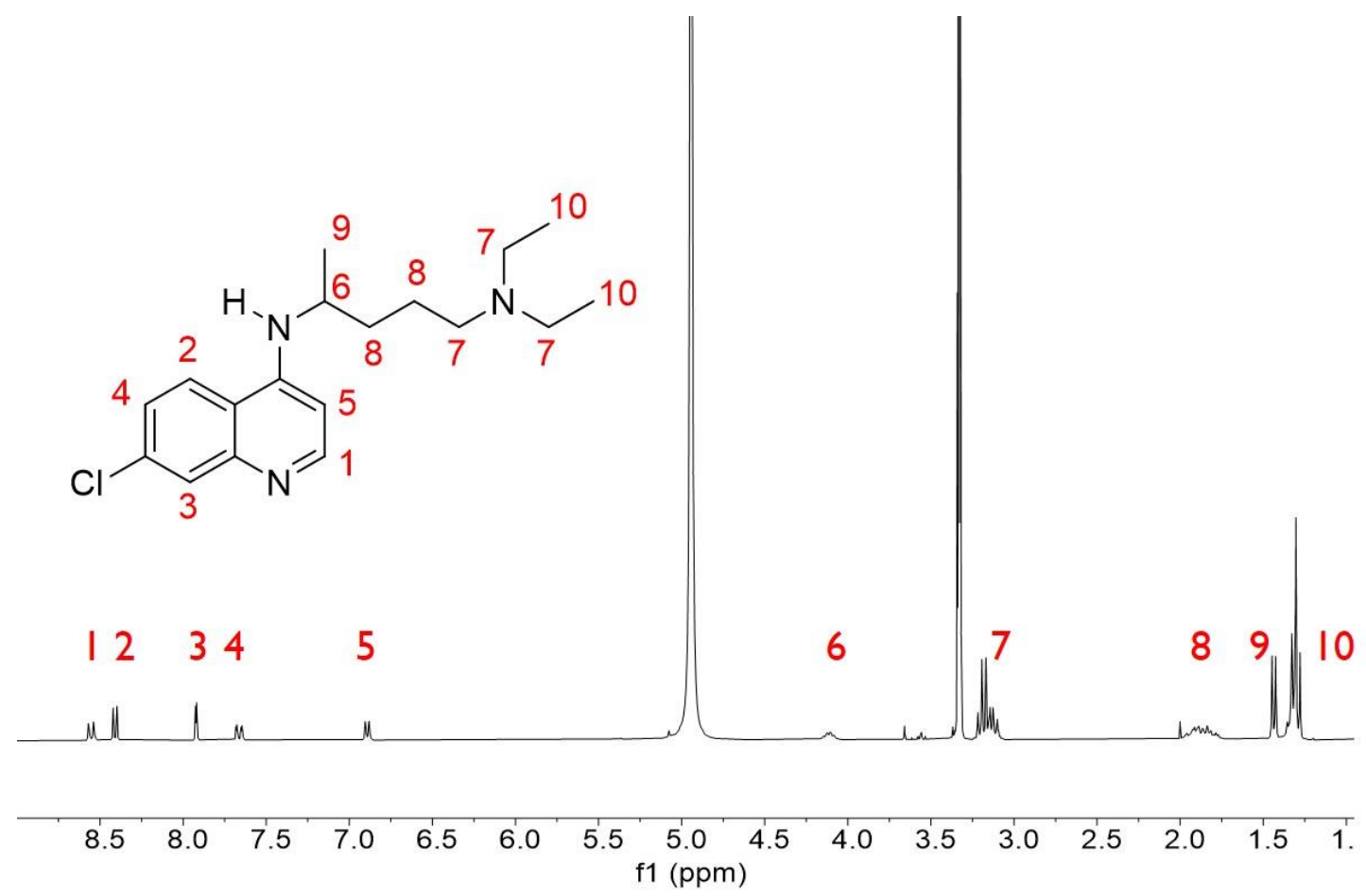




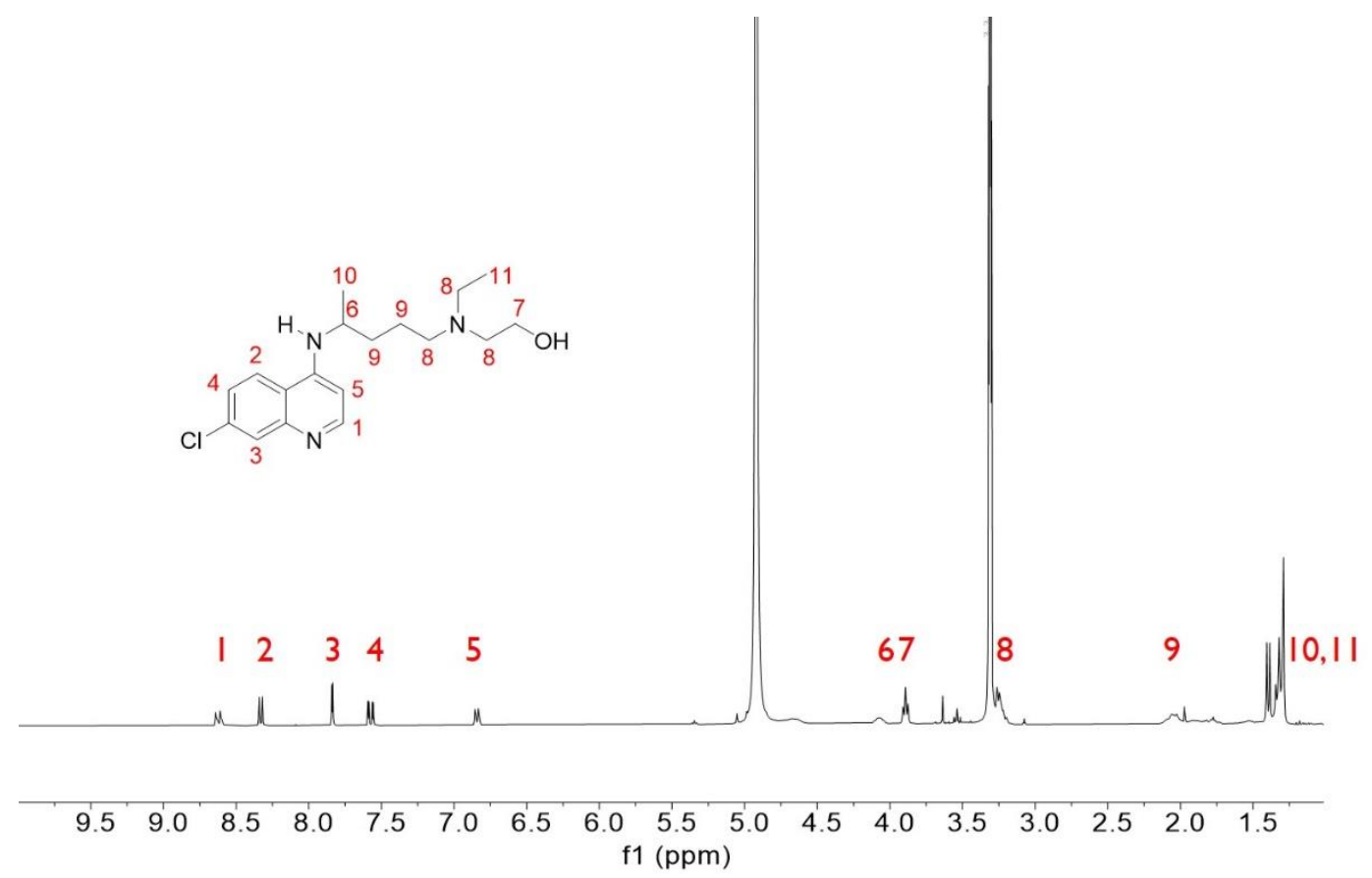

Figure 3. Chloroquine (upper structure) and Hydroxychloroquine (low structure) molecular structure and those normal NMR signals.

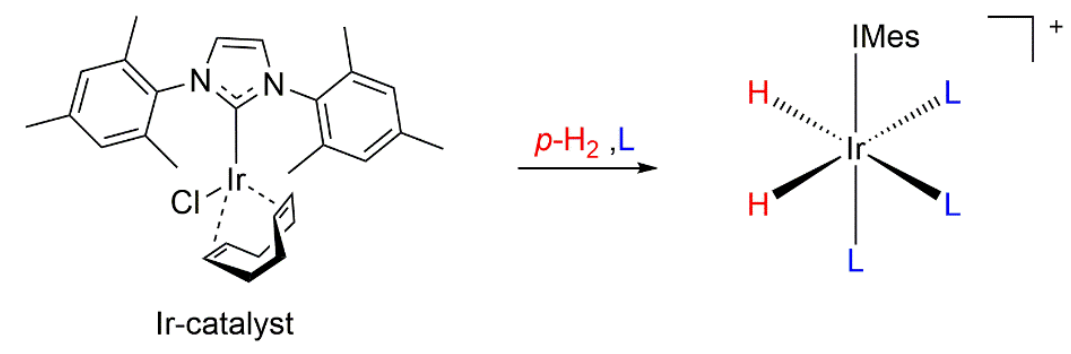<smiles>CCN(CC)CCCC(C)Nc1cc[n+](C(C)(C)C)c2cc(Cl)ccc12</smiles>

Figure 4. Ir-catalyst and Chloroquine (hydroxychloroquine) / Favipiravir complex structures for SABRE in methanol. 
Interestingly, the polarization transfer from para-hydrogen to chloroquine (hydroxychloroquine) was noteworthy as the polarization transfer occurred across a long distance of 11 bonds (from nitrogen to hydrogen number 10). This is the first observation of such a long-range hyperpolarization via SABRE (Figure 5).<smiles>CCN(CCCC(C)Nc1ccnc2cc(Cl)ccc12)C(C)C</smiles>

\section{Hyperpolarization}

12345

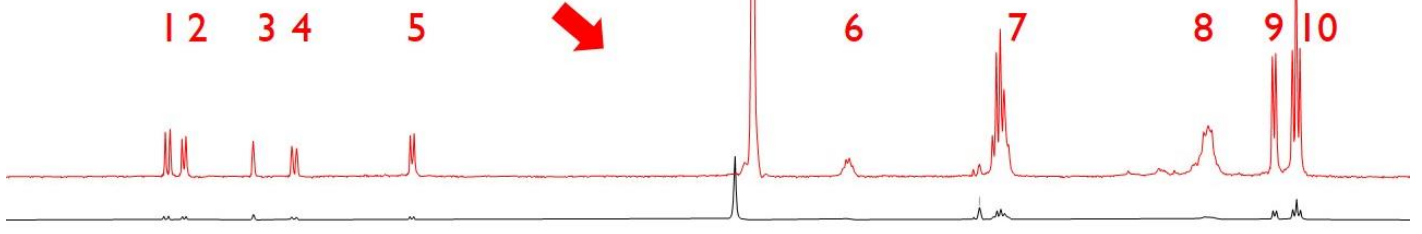

\begin{tabular}{llllllllllllllllll}
\hline .5 & 9.0 & 8.5 & 8.0 & 7.5 & 7.0 & 6.5 & 6.0 & $\begin{array}{c}5.5 \\
\mathrm{f} 1(\mathrm{ppm})\end{array}$ & $\begin{array}{c}5.0 \\
(\mathrm{ppm})\end{array}$ & 4.5 & 4.0 & 3.5 & 3.0 & 2.5 & 2.0 & 1.5 & 1.0
\end{tabular}<smiles>O=C(CCCCO)Nc1ccnc2cc(Cl)ccc12</smiles>

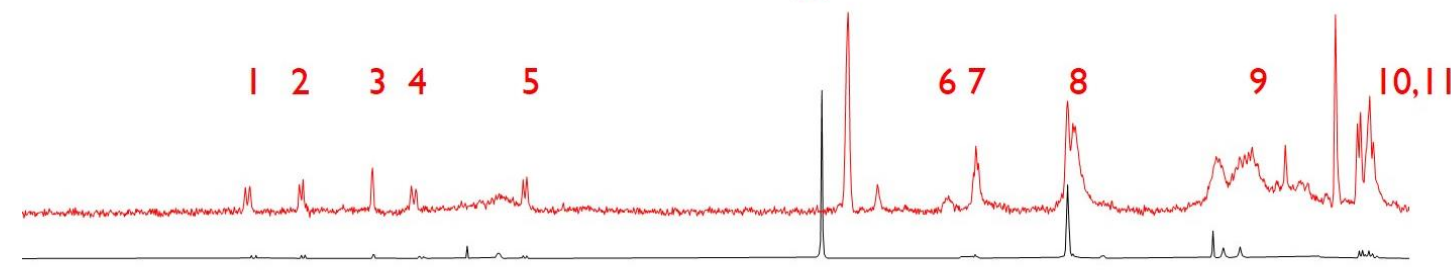

$\begin{array}{lllllllllllllllllll}10.0 & 9.5 & 9.0 & 8.5 & 8.0 & 7.5 & 7.0 & 6.5 & \underset{f 1}{6(\mathrm{ppm})} & 6.0 & 5.5 & 5.0 & 4.5 & 4.0 & 3.5 & 3.0 & 2.5 & 2.0 & 1.5\end{array}$

Figure 5. Hyperpolarized signals from chloroquine (upper structure) and hydroxychloroquine (low structure) after SABRE in the presence of a $90 \mathrm{G}$ and $110 \mathrm{G}$ magnetic field, respectively. 
To understand the mechanisms at play, the enhancement of chloroquine (hydroxychloroquine) by hyperpolarization was measured by changing the magnetic field in the polarization transfer period (Figure 6).
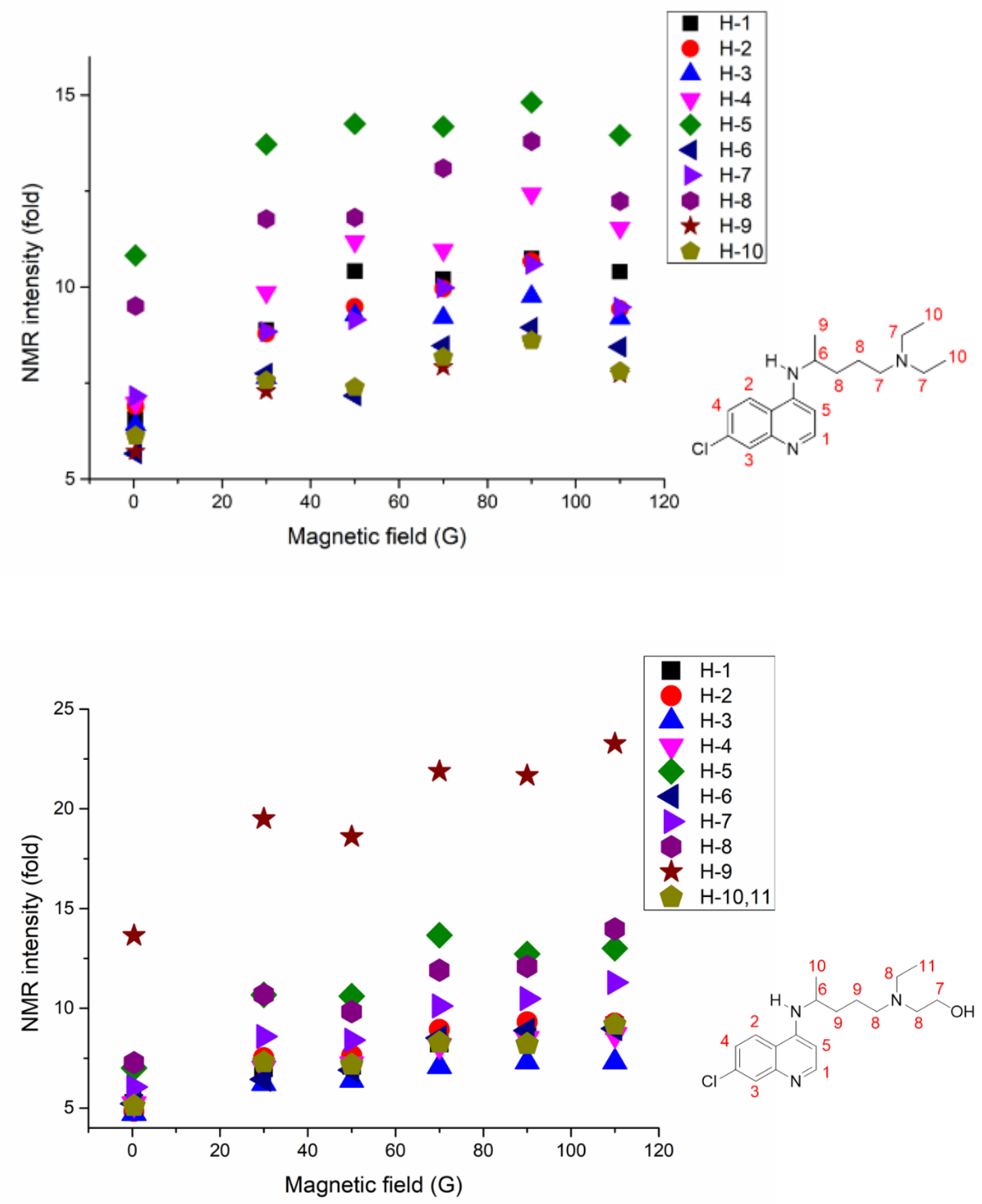

Figure 6. Amplification number of protons from hyperpolarized chloroquine (hydroxychloroquine) structure. 
Although the degree of polarization enhancement was similar, the protons in carbon $(\mathrm{H}-9$ in hydroxychloroquine and $\mathrm{H}-8$ in chloroquine) had the highest and the second highest enhancement (respectively); this differs greatly from the nitrogen in the quinoline moiety. This difference may stem from the direct polarization transfer from hydride, which bonds to the Ircatalyst to H-9 (hydroxychloroquine), H-8 (chloroquine) by dipolar coupling or polarization transfer from H-5 to H-9/8 by dipolar coupling. This requires further detailed future research. Similarly, the H-5 proton in quinoline on both structures showed high enhancement. These results indicate that the mechanism of polarization transfer is dependent on even a small change in structure and solubility.

Despite its relatively small polarization enhancement, it is assumed that it will increase through the use of higher para-hydrogen concentrations and partial pressure. In doing this, the enhancement may drastically improve. As Figure 6 indicates, polarization maximized around $70 \mathrm{G}$, exhibiting a similar trend as previous reports on polarization transfer mechanisms. ${ }^{31}$

Ritonavir/Lopinavir SABRE Ritonavir/Lopinavir does not contain any well-known functional groups in the structure, which can be harnessed to efficiently undergo polarization transfer from para-hydrogen. In particular, lopinavir does not contain $\mathrm{sp}^{2}$ nitrogen in the structure, which has been widely used for polarization transfer. However, both structures contain the carbonyl group, which can transfer polarization from para-hydrogen to the carbonyl group with the ester group/amide group in the neighborhood. A recent study demonstrated that the carbonyl group can bind to the Ir-catalyst, and polarization can be transferred to pyruvate. ${ }^{34}$ Moreover, we demonstrated that the Ir-catalyst was binding with carbonyl and phenyl ether by detecting the chemical shift of lopinavir protons of $\mathbf{1}$ (Figure 7) after the complex was formed. 

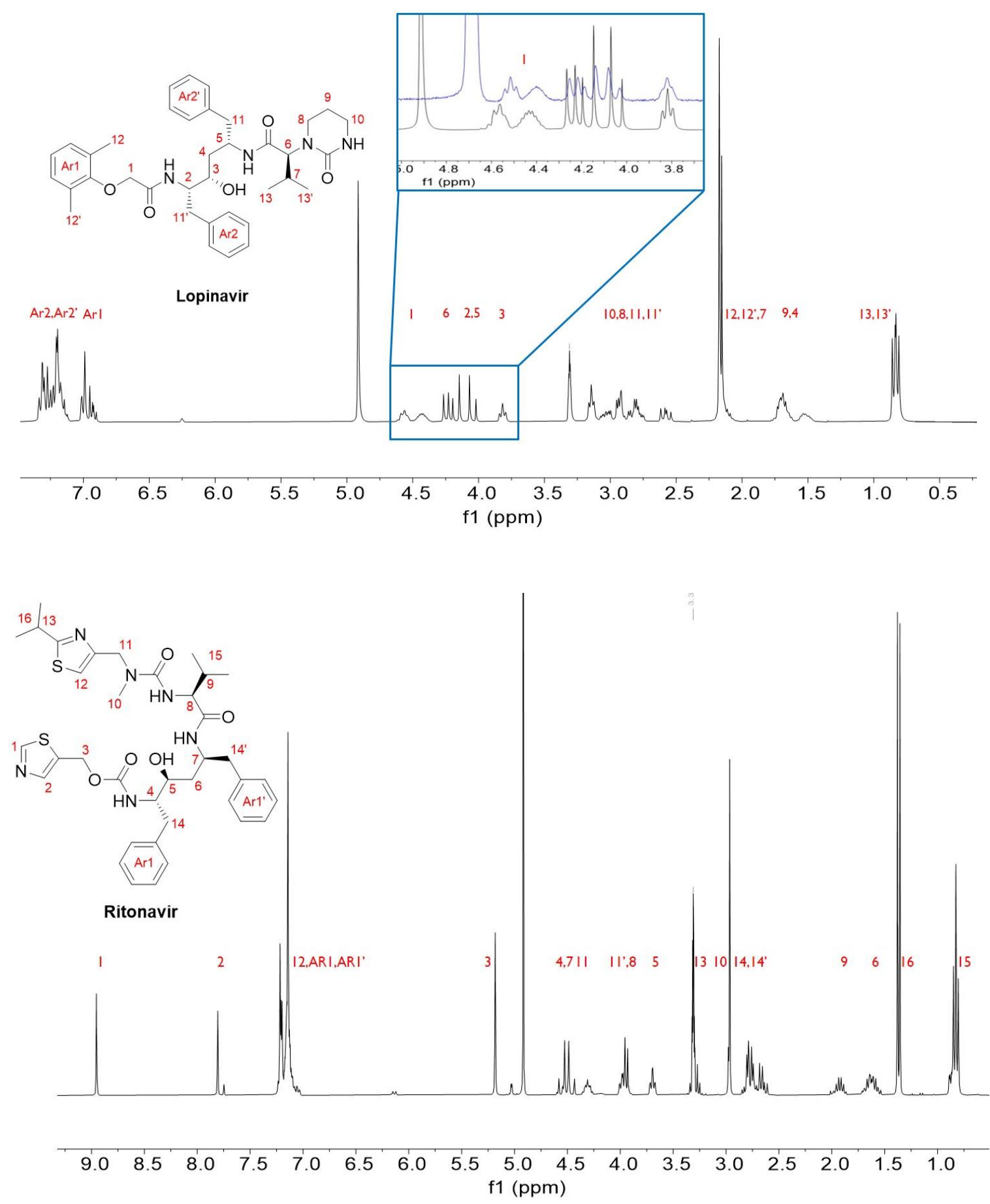

Figure 7. Lopinavir (upper structure) and ritonavir (low structure) molecular structures and their respective normal NMR signals. The lopinavir proton of $\mathbf{1}$ shifted after binding with the Ir-catalyst (blue spectrum).

This is the first case in which a binding trend has been identified and, interestingly, polarization was transferred to nearly all protons of lopinavir through this binding site. 
The polarization transfer from para-hydrogen to ritonavir/lopinavir is noteworthy as the polarization transferred across a long distance of more than 12 bonds in lopinavir. This is also the first case wherein extremely long-range hyperpolarization via SABRE was observed after binding with the carbonyl group (Figure 8).
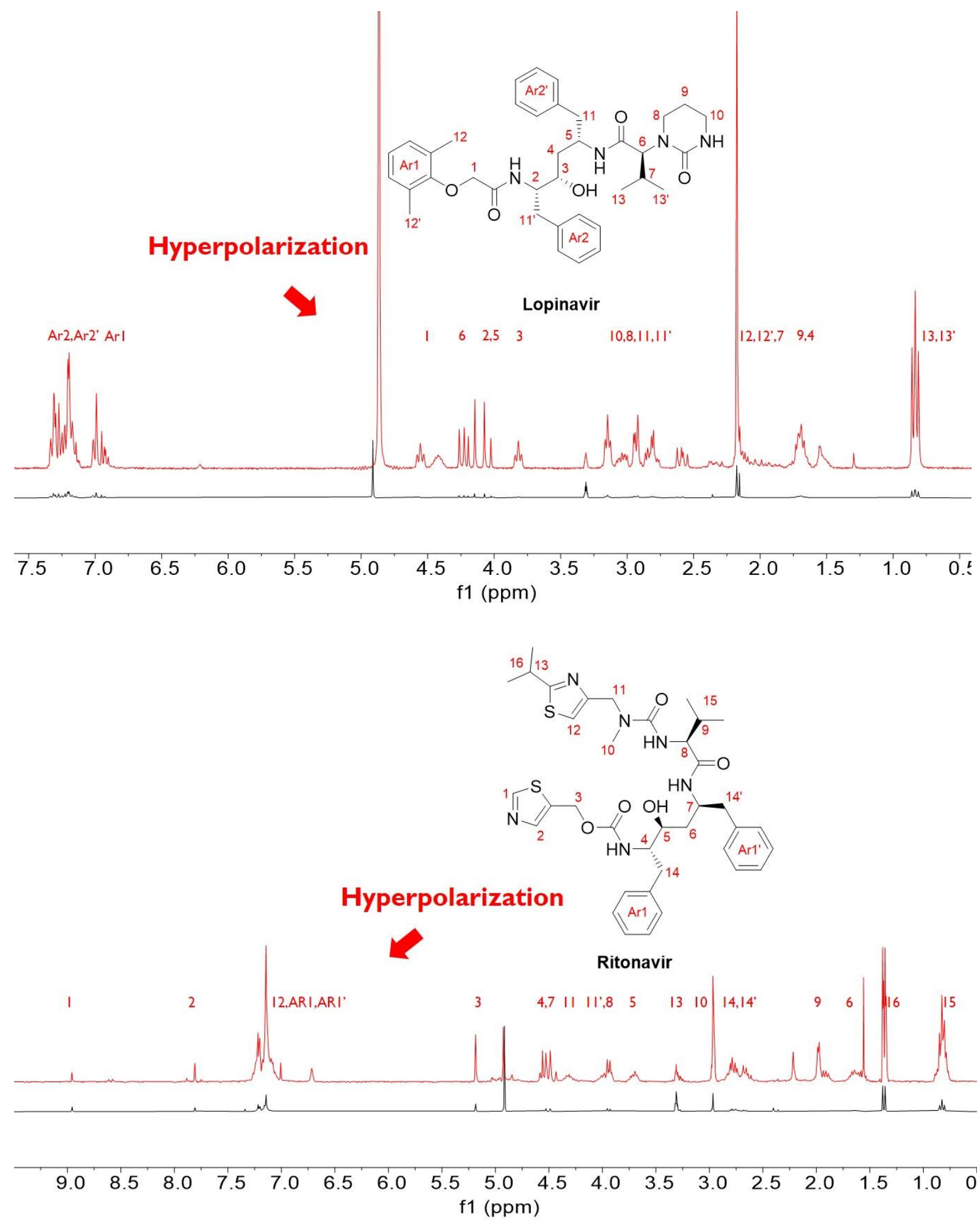
Figure 8. Hyperpolarized signal from lopinavir (upper spectrum) and ritonavir (low spectrum) after SABRE in the presence of a $70 \mathrm{G}$ magnetic field.

The enhancement of the proton NMR signal on lopinavir by hyperpolarization was measured by changing the magnetic field in the polarization transfer period to understand the SABRE mechanism (Figure 9).
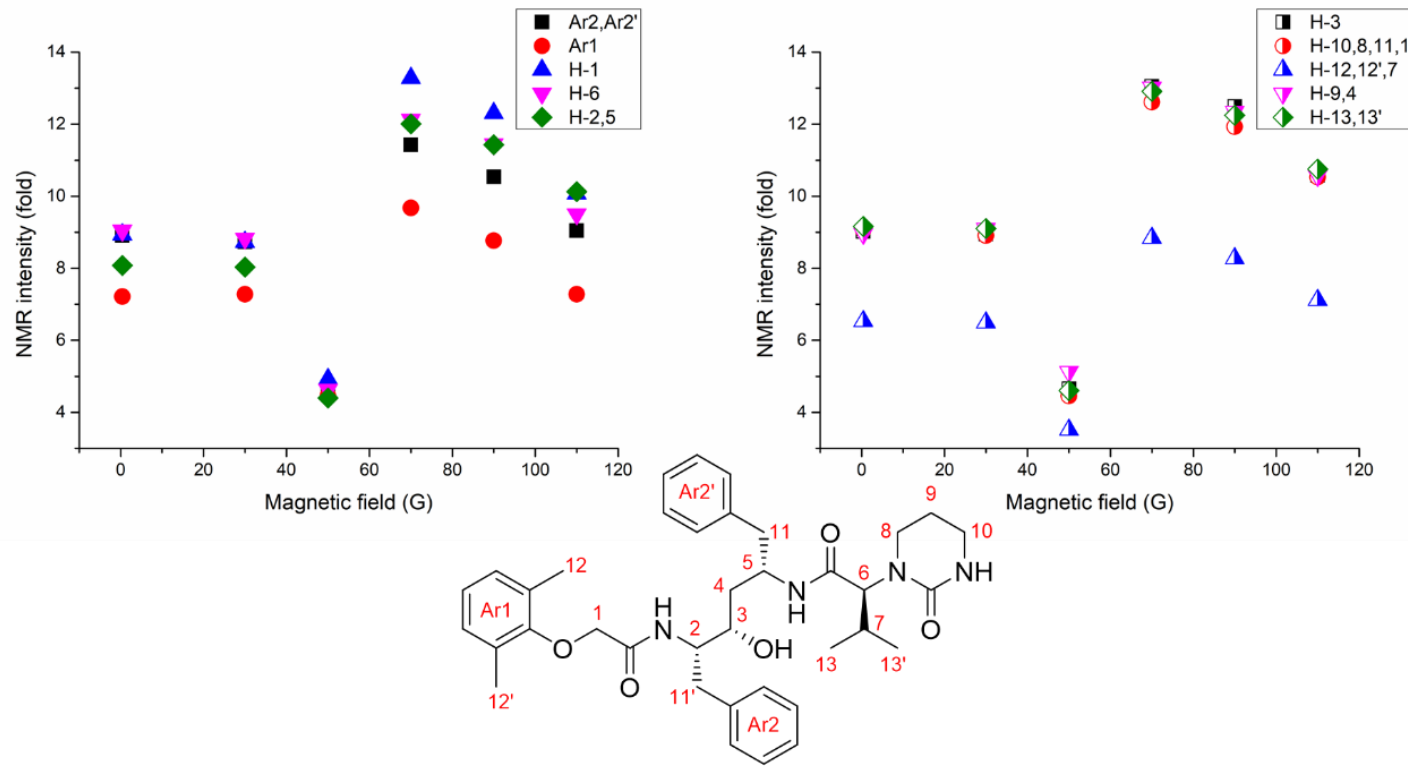

Lopinavir

Figure 9. Amplification number of protons from hyperpolarized lopinavir structure. 


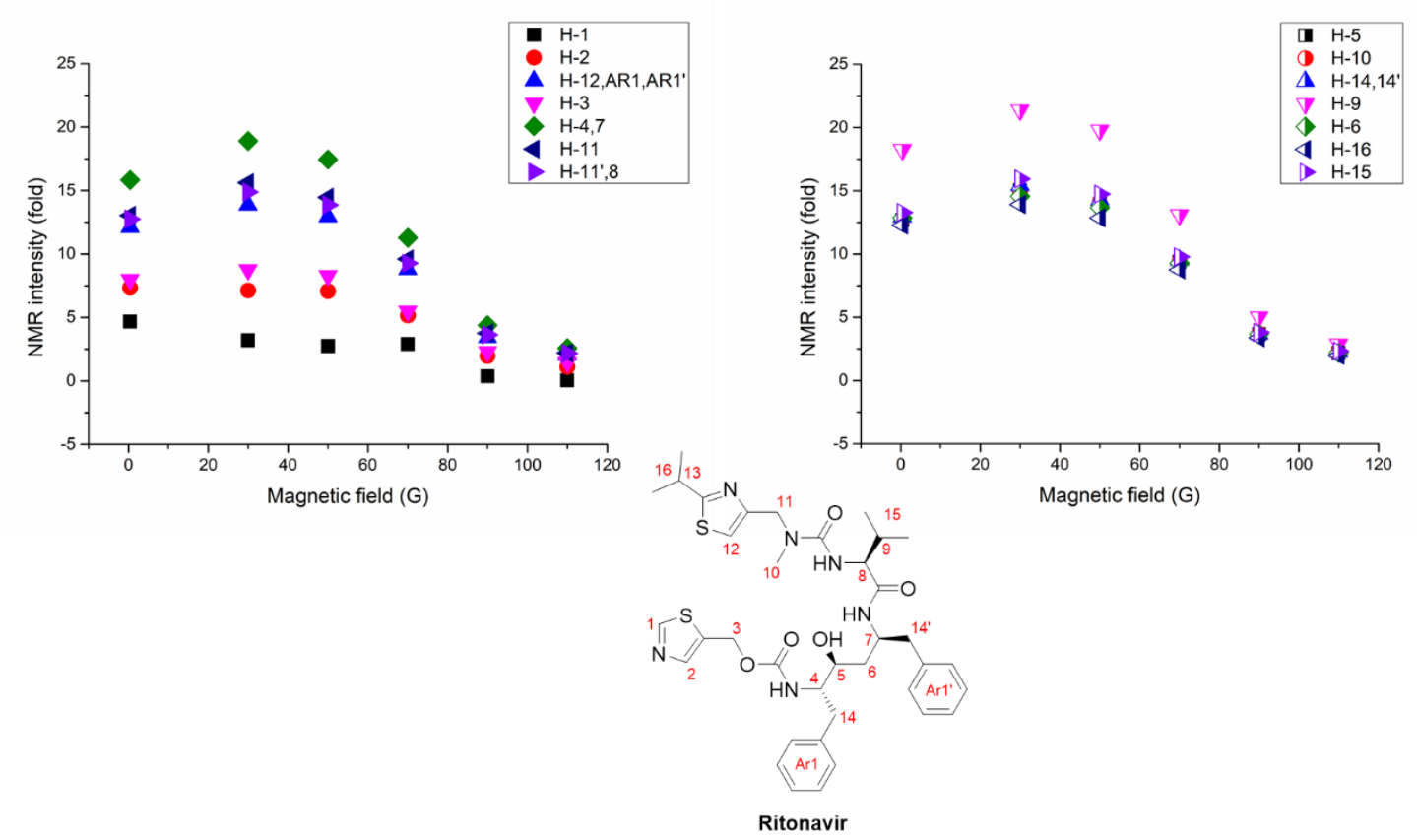

Figure 10 Amplification number of protons from hyperpolarized ritonavir structure.

This enhancement was also relatively small, however, it is expected to increase when higher para-hydrogen concentrations and partial pressures are used. As Figure 9 shows, polarization was maximized around $70 \mathrm{G}$, which exhibits a similar trend to previous reports on polarization transfer mechanisms, including chloroquine. Interestingly, H-8 and H-10 had the highest polarization enhancement during SABRE. This may be attributed to the dipolar coupling, polarization transfer through the space, and the SABRE-RELAY mechanism. These require further detailed studies in the future. ${ }^{35}$.

In terms of the results associated with ritonavir SABRE, the 30-50 G was the optimum external magnetic field to match the polarization transfer (Figure 10). This is a low field compared to results of the previous antiviral drug SABRE. This comparison reflects that the optimum magnetic field for polarization transfer is dependent on the binding site with the Ir-catalyst and the structure, which can induce different scalar coupling constants. As such, we need to optimize the magnetic field to yield the maximum polarization enhancement in different materials such as the target materials, solvent, and catalysts. It was difficult to identify the binding site of ritonavir with the Ir-catalyst as there were several possible binding sites to consider. We observed a small chemical shift in almost all protons of ritonavir after mixing with the Ir-catalyst. This implies that there "may be more than one binding site for ritonavir with the Ir-catalyst. This finding is supported as there are many possible binding sites such as two thiazole moieties and three carbonyl sites. Despite the presence of several binding sites in 
ritonavir, note that hyperpolarization occurred on all protons in the structure and its enhancement of polarization was almost identical in the higher field. This offers a good explanation as to the presence of several binding sites with the Ir-catalyst. Ritonavir's polarization characteristics in the whole structure was only attributable to long-range polarization transfer, the main polarization transfer mechanism for SABRE.

These long-range polarization transfers imply its potential use with hyperpolarized signals in NMR/MRI. This may provide a better understanding of molecular dynamics with targeted proteins and pharmacokinetics. Importantly, in future studies, it is anticipated that it may be used for tracking the hyperpolarized signal through MRI. Examples of this include isotope labeling (such as carbon to ${ }^{13} \mathrm{C}$ isotope and nitrogen into ${ }^{15} \mathrm{~N}$ ), in the structures of those studied drugs, which may have a long T1 time. This is not only because of the lower gyromagnetic ratio, but also because of its smaller relaxation effect from the Ir-catalyst by short binding time due to its relatively unstable complex structure.

\section{Conclusions}

Antiviral drugs, such as chloroquine, hydroxychloroquine, ritonavir, lopinavir, and favipiravir show promise as drug candidates in response to the COVID-19 pandemic situation. Spin hyperpolarization on such well-known drugs may open new opportunities in the diagnosis and biomedical research of COVID-19 via MRI and pharmacodynamics, metabolomics, and binding dynamics with proteins. This can be achieved by using enhanced signal intensity in NMR/MRI. Furthermore, this SABRE-based hyperpolarization study based on these high molecular weight structures, was not previously conducted due to the special polarization transfer mechanism. In this study, high molecular weight chloroquine, hydroxychloroquine, ritonavir, lopinavir, and favipiravir were successfully tested for their SABRE-based hyperpolarization. Polarizations over long distances were detected; a noteworthy result for future research, as this technique may be used for other materials. Each polarization transfermaximized external magnetic field was slightly different, clearly implying that each structure has different optimal polarization transfer matching conditions. Therefore, matching the magnetic field should be controlled to obtain efficient polarization enhancement in each structure. Importantly, this method can be used to monitor a drug's distribution and activity in vivo by MRI. It can be harnessed to further investigate the molecular interaction of drug 
candidates with key proteins and unveil unknown activity on COVID-19, including pharmacokinetics.

The polarization of the proton may be transferred to any other isotope nuclei using pulse sequence, field cycling, and matching of the spin energy. In future, this will address the ultimate objective of obtaining hyperpolarized antiviral drugs to study their activity on COVID-19 with a sufficiently long time of T1.

This study was unable to shed significant light on the real applications of the treatment of COVID-19. However, many related studies have reported applications in drug discovery, detecting tumors in vivo, and polarization transfer into many other isotopes along with pulse sequence development. Future work on isotope labeling, and further polarization transfer on long T1 time nuclei including clinical perspectives may open up new opportunities for overcoming this global pandemic. 


\section{Acknowledgement}

This work was supported by the National Research Foundation of Korea (NRF) grant funded by the Korean government (MSIT) (No. 2020R1C1C1007888).

\section{References}

1. Huang, C. et al. Clinical features of patients infected with 2019 novel coronavirus in Wuhan, China. Lancet 395, 497-506 (2020).

2. Wang, M. et al. Remdesivir and chloroquine effectively inhibit the recently emerged novel coronavirus (2019-nCoV) in vitro. Cell Res. 30, 269-271 (2020).

3. Yan, Y. et al. Anti-malaria drug chloroquine is highly effective in treating avian influenza A H5N1 virus infection in an animal model. Cell Res. 23, 300-302 (2013).

4. Yan, Y. et al. Anti-malaria drug chloroquine is highly effective in treating avian influenza A H5N1 virus infection in an animal model. Cell Res. 23, 300-302 (2013).

5. Vincent, M. J. et al. Chloroquine is a potent inhibitor of SARS coronavirus infection and spread. Virol. J. 2, 69 (2005).

6. Savarino, A. \& Shytaj, I. L. Chloroquine and beyond: Exploring anti-rheumatic drugs to reduce immune hyperactivation in HIV/AIDS. Retrovirology 12, 51 (2015).

7. Mackenzie, A. H. Dose refinements in long-term therapy of rheumatoid arthritis with antimalarials. Am. J. Med. 75, 40-45 (1983).

8. Zheng, J. SARS-CoV-2: an Emerging Coronavirus that Causes a Global Threat. Int. J. Biol. Sci. 16, 1678-1685 (2020).

9. Yao, T., Qian, J., Zhu, W., Wang, Y.\& Wang, G. A systematic review of lopinavir therapy for SARS coronavirus and MERS coronavirus - A possible reference for coronavirus disease-19 treatment option. J. Med. Virol. jmv.25729 (2020). doi:10.1002/jmv.25729

10. Wang, Z., Chen, X., Lu, Y., Chen, F. \& Zhang, W. Clinical characteristics and therapeutic procedure for four cases with 2019 novel coronavirus pneumonia receiving combined Chinese and Western medicine treatment. Biosci. Trends 14, 64-68 (2020).

11. Lim, J. et al. Case of the index patient who caused tertiary transmission of coronavirus disease 2019 in Korea: The application of lopinavir/ritonavir for the treatment of COVID-19 pneumonia monitored by quantitative RT-PCR. J. Korean Med. Sci. 35, (2020). 
12. Kim, J. Y. et al. The first case of 2019 novel coronavirus pneumonia imported into korea from wuhan, china: Implication for infection prevention and control measures. $J$. Korean Med. Sci. 35, e61 (2020).

13. Goldhill, D. H. et al. The mechanism of resistance to favipiravir in influenza. Proc. Natl. Acad. Sci. U. S. A. 115, 11613-11618 (2018).

14. Hayden, F. G. \& Shindo, N. Influenza virus polymerase inhibitors in clinical development. Curr. Opin. Infect. Dis. 32, 176-186 (2019).

15. Chen, C. et al. Favipiravir versus Arbidol for COVID-19: A Randomized Clinical Trial. medRxiv 2020.03.17.20037432 (2020). doi:10.1101/2020.03.17.20037432

16. Tanner, C. P. N. et al. Selective hyperpolarization of heteronuclear singlet states via pulsed microtesla SABRE. J. Chem. Phys. 151, 044201 (2019).

17. Barskiy, D. A. et al. Over 20\% 15N Hyperpolarization in under One Minute for Metronidazole, an Antibiotic and Hypoxia Probe. J. Am. Chem. Soc. 138, 8080-8083 (2016).

18. Glachet, T. et al. Iodonitrene in Action: Direct Transformation of Amino Acids into Terminal Diazirines and 15N2-Diazirines and Their Application as Hyperpolarized Markers. J. Am. Chem. Soc. 141, 13689-13696 (2019).

19. Iali, W., Rayner, P. J. \& Duckett, S. B. Using parahydrogen to hyperpolarize amines, amides, carboxylic acids, alcohols, phosphates, and carbonates. Sci. Adv. 4, (2018).

20. Ariyasingha, N. M. et al. Quasi-resonance fluorine-19 signal amplification by reversible exchange. J. Phys. Chem. Lett. 10, 4229-4236 (2019).

21. Olaru, A. M. et al. Using signal amplification by reversible exchange (SABRE) to hyperpolarise 119Sn and 29Si NMR nuclei. Chem. Commun. 52, 14482-14485 (2016).

22. Rayner, P. J., Richardson, P. M. \& Duckett, S. B. The Detection and Reactivity of Silanols and Silanes Using Hyperpolarized 29 Si Nuclear Magnetic Resonance. Angew. Chemie 132, 2732-2736 (2020).

23. Iali, W. et al. Hyperpolarising Pyruvate through Signal Amplification by Reversible Exchange (SABRE). Angew. Chemie - Int. Ed. 58, 10271-10275 (2019).

24. Richards, J. E. et al. Using hyperpolarised NMR and DFT to rationalise the unexpected hydrogenation of quinazoline to 3,4-dihydroquinazoline. Chem. Commun. 54, 10375-10378 (2018).

25. Ratajczyk, T. et al. NMR Signal Enhancement by Effective SABRE Labeling of Oligopeptides. Chem. - A Eur. J. 21, 12616-12619 (2015).

26. Svyatova, A. et al. 15N MRI of SLIC-SABRE Hyperpolarized 15N-Labelled Pyridine and Nicotinamide. Chem. - A Eur. J. 25, 8465-8470 (2019).

27. Robertson, T. B. R. et al. Hyperpolarization of Pyridyl Fentalogues by Signal Amplification By Reversible Exchange (SABRE). ChemistryOpen 8, 1375-1382 (2019). 
28. Linnik, I. V., Rayner, P. J., Stow, R. A., Duckett, S. B. \& Cheetham, G. M. T. Pharmacokinetics of the SABRE agent 4,6-d 2 -nicotinamide and also nicotinamide in rats following oral and intravenous administration. Eur. J. Pharm. Sci. 135, 32-37 (2019).

29. Jeong, K., Min, S., Chae, H. \& Namgoong, S. K. Monitoring of hydrogenation by benchtop NMR with parahydrogen-induced polarization. Magn. Reson. Chem. 57, 4448 (2019).

30. Eshuis, N. et al. Determination of long-range scalar $1 \mathrm{H}-1 \mathrm{H}$ coupling constants responsible for polarization transfer in SABRE. J. Magn. Reson. 265, 59-66 (2016).

31. Atkinson, K. D. et al. Spontaneous transfer of Parahydrogen derived spin order to pyridine at low magnetic field. J. Am. Chem. Soc. 131, 13362-13368 (2009).

32. Adams, R. W. et al. Reversible interactions with para-hydrogen enhance NMR sensitivity by polarization transfer. Science (80-. ). 323, 1708-1711 (2009).

33. Daniele, V., Legrand, F. X., Berthault, P., Dumez, J. N. \& Huber, G. Single-Scan Multidimensional NMR Analysis of Mixtures at Sub-Millimolar Concentrations by using SABRE Hyperpolarization. ChemPhysChem. 16, 3413-3417 (2015).

34. Tickner, B. J. et al. Optimisation of pyruvate hyperpolarisation using SABRE by tuning the active magnetisation transfer catalyst. Catal. Sci. Technol. 10, 1343-1355 (2020).

35. Iali, W., Rayner, P. J. \& Duckett, S. B. Using parahydrogen to hyperpolarize amines, amides, carboxylic acids, alcohols, phosphates, and carbonates. Sci. Adv. 4, eaao6250 (2018). 